MSS, Vol. 5, No. 2, July - December 2006, pp. 57-70

ISSN 0975-3311

https:/ / doi.org/ 10.12725/ mjs.9.6

\title{
INVESTIGATION ON LIQUID CRYSTALLINE SYSTEMS
}

\author{
Manu S*, Fyna Francis** \& Tobin Scaria***
}

\begin{abstract}
The results of experiments carried out by us on two samples exhibiting a macroscopic helical structure. While one of them shows a direct fransition from the chiral orientationally ordered fluid (referred to an $\mathrm{N}^{*}$ phase) to a phase with one dimensional layer structure, the second sample exhibits a liquid crystal analog of the Abrikosov flux lattice of super conductors, between the $N^{*}$ and layered phase. The chapter compares and contrasts the differences in the behoviour of the two compounds.
\end{abstract}

\section{Introduction}

The Twist Grain Boundary phase, it was discovered in 1988 and has attracted significant attention since then. In the following we give a brief description of the

* No.43, $3^{\text {r }}$ main road, Kengeri Upanagar, Bangalore 560 060. Ph. 080-57680470.

Email: manuchrist@gmail.com

** Moliekal Koonan (H), Shantinagar, Puthenchira (E), Thrissur, Kerala 680 682. Ph. $0480-2893963$ Email: fynofrancis@yahoo.co.uk

***Kuzhippallil, Koomanthode P.O, Kannur, Kerala 670 704. Ph. 0490-2421312.

Email: tobin_scaria@yahoo.com 
structure of this exotic phase and its importance. Chiral liquid crystals have the tendency to form a cholesteric like helical director field. On the other hand, the molecular interactions may favor a smectic layer structure. However, it is impossible to realize a continuous structure which exhibits both a cholesteric director field and a smectic layer structure at the same time. The competition between these two structural features can result in frustrated structures containing a regular lattice of grain boundaries which in turn consist of a lattice of screw dislocations. This defect structure exhibits an interesting theoretical analogy to the flux line lattice which occurs in the abrikosov phase of type II superconductors. However, the range of parameters determining the structure is larger in liquid crystals than in superconductors. Thus a large variety of new phases, such as $T G B_{A^{\prime}} T G B_{c^{\prime}} T G B_{c^{*}}$ have been predicted and the subscript indicates the types of the underlying smectic structure and or experimentally observed.

Twisted grain boundary (TGB) phases usually appear in the temperature range between a cholesteric phase with short pitch and a smectic phase, typically smectic $\mathrm{A}$ or smectic $\mathrm{C}^{*}$. One of their remarkable properties is the selective reflection of circularly polarized light. This feature shows that the director field has a helical

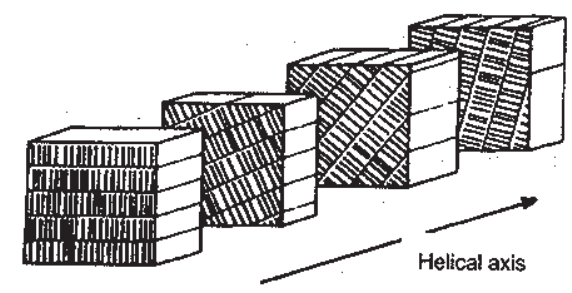

Figure 1.1: Structure of the $T G B_{A}$ phase which shows a local smectic order and a helical director field at the same time.

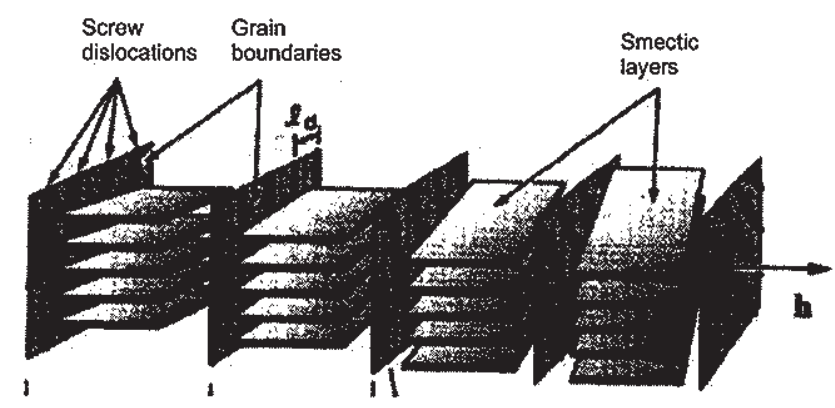

Figure 1.2: Another schematic view of the $T G B_{A}$ phase. 
structure similar to the cholesteric phase. On the other hand, $X$-ray investigations of TGB phases indicate a layer structure as occurring in smectic phases. Chirality of the system is an essential precondition for the occurrence of TGB phases. In mixtures of chiral and nonchiral components, the concentration of the chiral component must exceed a critical value in order to lead to TGB structures.

\section{The Analogy between TGB Phases and Superconductors}

The existence of the $T G B_{A}$ phase characterized both by local smectic layering and by an average twist of the direction of molecular alignment characteristic of $a$ chiral nematic phase. The theoretical basis for this prediction was the de Gennes model for the nematic to smectic A transition, which establishes a strong analogy between this transition and the normal to superconducting transition in metals. The nature of the normal to superconducting transition in the presence of an external magnetic field depends on the Ginzburg parameter $x=\lambda / \xi$ specifying the ratio of the magnetic penetration depth $\lambda$ to the coherence length $\xi$.
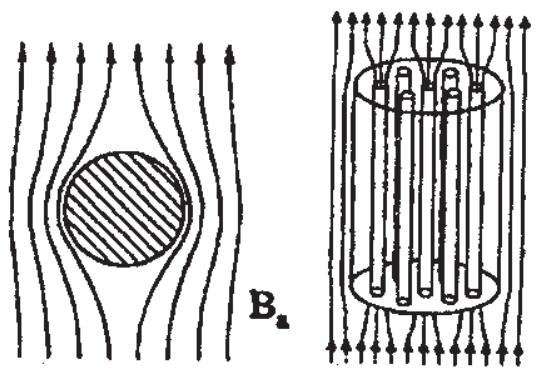

Figure 1.3: Shows the Meissner effect and Abrikosov flux lattice for type II superconductors

For type I systems with $\kappa<1 / 2^{1 / 2}$, there is a first - order transition from a normal metal to a Meissner superconducting phase in which the magnetic flux is totally expelled, whereas for type-ll systems with $k<1 / 2^{1 / 2}$, there is a second-order transition in mean field theory from the normal metal to the Abrikosov superconducting phase in which there is a partial penetration of magnetic flux in the form of a regular lattice of vortices. The latter transition is followed, upon further decrease in temperature, by a second order transition to the Meissner phase. The twist grain boundary phase is the analog of the Abrikosov phase in superconductors with molecular chirality the analog of the external magnetic field. Figure 1.3 describes 
the Meissner effect in superconductors and Abrikosov flux lattice in type II superconductors where the external field is not completely expelled but penetrates in the superconductor along parallel tubes which form a regular lattice.

In a manner similar to the expulsion of the magnetic field in the superconducting phase, helical structure (rather the twist of the director) is expelled from the layered smectic A phase in a type I system. Thus, the $T G B_{A}$ phase in which the twist creating the grain boundaries penetrates the smectic structure periodically completes the analogy between the liquid crystal and superconducting systems.

\section{Experiment}

The experiment aimed at investigating one partial property of liquid crystals exhibiting a supromolecular chiral structure, viz., the pitch of the helix, its thermal variation and the associated critical phenomena.

\section{Materials}

Experiments have been carried out on the two following different liquid crystalline compounds,

\section{Compound 1}

Cholesteryl nonanoate, a commercially available monomeric compound.

\section{Compound 2}

Cholesterol ester based dimesogen, synthesized in CLCR, in which the cholesterol ester part was tethered to another mesogenic unit through a flexible alkyl spacer.

The phase sequence and the transition temperatures obtained in the cooling mode for the two compounds are given below.

\section{Compound 1}

Isotropic $85^{\circ} \mathrm{C}$ chiral nematic $81.5^{\circ} \mathrm{C}$ smectic $\mathrm{A} 73.1^{\circ} \mathrm{C}$

\section{Compound 2}

Isotropic $190^{\circ} \mathrm{C}$ chiral nematic $170.4^{\circ} \mathrm{C} \quad \mathrm{TGB}_{\mathrm{A}} \quad 161.2^{\circ} \mathrm{C}$ 


\section{Methods}

The aim of the investigations was to determine the pitch of the helix using the selective reflection method. In a small wavelength range about $\lambda_{0}$, an incident polychromatic light beam parallel to the helix axis is split into its circularly polarized components, one of which is simply transmitted whereas the other is totally reflected; the rotation of the reflected circularly polarized light is in conformity with the screw sense of the helix. When the beam is incident normally, maximum reflection occurs when the wavelength of the incident light satisfies the Bragg condition

$$
\lambda_{0}=\mathrm{n}_{\mathrm{ovg}} \mathrm{P}
$$

where $P$ is the itch length of the helix and $n_{\text {avg }}=\left(n_{0}+n_{e}\right) / 2$, the average refractive index. The beam which does not satisfy $\lambda \stackrel{\text { avg }}{=} \lambda_{0}$ is simply transmitted. This is the selective reflection property. The bandwidth of the reflection $\Delta \lambda=\lambda_{0} \Delta n / n_{\text {ovg }}$ where $\Delta \mathrm{n} \sim 0.2$ is the birefringence, yielding $\Delta \lambda \sim 50 \mathrm{~nm}$. Such a periodic structure can be viewed as a $1-D$ photonic crystal with a band gap within which propagation of light is forbidden.

* As mentioned earlier, the geometry required to study the selective reflection property is the one in which the helical axis is normal to the substrate surface. To achieve this geometry we proceeded as follows

The sample cell used for the purpose was made from two flat glass plates. In order to realize uniform orientation of the liquid crystal molecules parallel to the substrate surface the glass plates were first thoroughly cleaned using a standard procedure and then were treated with an alignment promoter using a technique outlined below.

The cleaned glass plate was mounted in a programmable speed spin coating unit (PNNP). A suitable quantity of the pre-polymer solution was placed on the glass plate. The plate was spun at a speed 3500 RPM for about 30 seconds. This process results in uniform coating of a thin film of the pre-polymer. This step is followed by in-situ polymerization of the film by heating the coated glass plate at $300^{\circ} \mathrm{C}$ for $\sim 3$ hours. The polymer film was then buffed unidirectionally ensuring the proper filling of the sample in the cell.

After the polarizing microscopy observations were made to ascertain the nature of the mesophases as well as to determine the phase transition temperatures associated with the different phase transformations. 


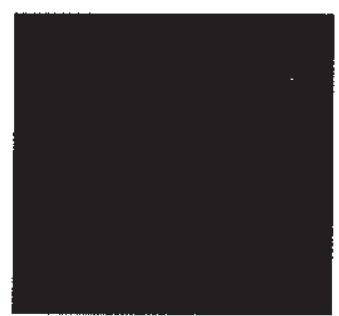

Figure 1.4: Texture showing the chiral nematic phase

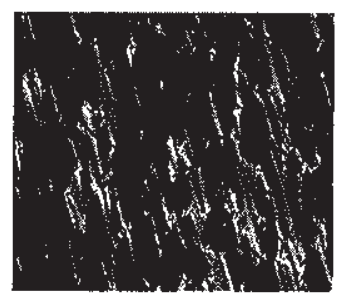

Figure 1.5: Focal conic texture of the smectic A phase

The experimental setup used for the selective reflection measurements is shown in figure 1.6. The sample cell was mounted in a hot stage (mettler FP 90) controlled by a programmable temperature controller (mettler FP 900). The hotstage was placed in the beam path of the UV - VIS - NIR Spectrometer (shimadzu).

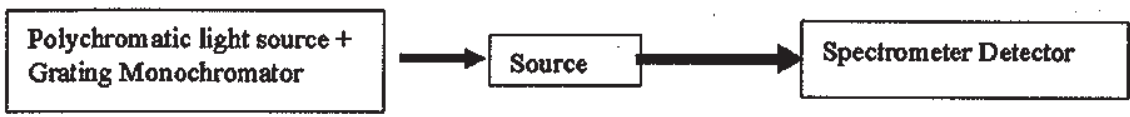

Figure 1.6: Experimental setup for selective reflection measurements.

The spectrometer consists of a continuous source $\left(\mathrm{D}_{2} /\right.$ tungsten) and the beam preparation was done by having a broadband grating monochromator. The spectral resolution of the beam was fixed at $2 \mathrm{~nm}$. The monochromator mounted on a turn table and therefore the built-in program of the spectrometer could select the beam wavelength and scan it over the desired range $200-700 \mathrm{~nm}$. The beam transmitted through the sample was detected using a photomultiplier, whose output was processed by digital electronics inside the spectrometer. The entire process of tuning the monochromator, detection and processing the signal was handled by a PC interfaced to the spectrometer. 


\section{Results and Discussion: compound 1}

Figures 1.4 and 1.5 show the optical textures observed in the two mesophases below the isotropic phase exhibiting the characteristic planar texture and the focal conic texture respectively establishing the phases to be chiral nematic and smectic A.

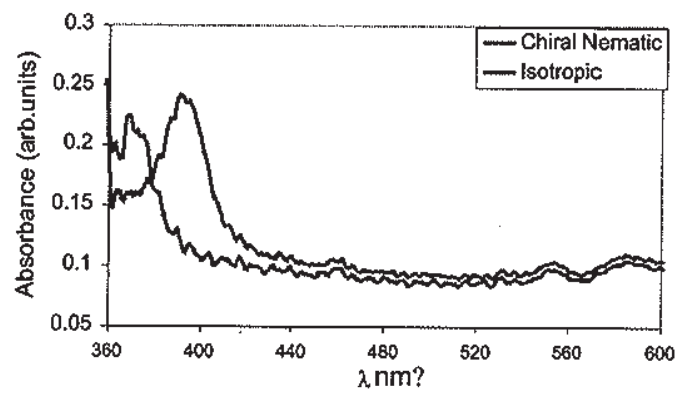

Figure 1.7: Spectroscopic profiles collected in the chiral nematic and isotropic phases

Figure 1.7 shows the new profile obtained in the chiral nematic and isotropic phases. In the isotropic phase, the absorption is very weak with hardly a variation as a function of wavelength. In contrast the profile in the chiral nematic exhibits a strong peak. The point as which the maximum absorption occurs is $\lambda_{0}$, the selective reflection wavelength.

Such profiles at $0.5^{\circ} \mathrm{C}$ intervals over the temperature range $83-74^{\circ} \mathrm{C}$ in the chiral nematic phase. In each case $\lambda_{0}$ was determined and is tabulated in table 1.1.

Table 1.1: Temperature dependence of the selective reflection wavelength $\lambda_{0}$ for compound 1 .

\begin{tabular}{cc}
\hline$T\left({ }^{\circ} \mathrm{C}\right)$ & $\lambda(\mathrm{nm})$ \\
\hline 83 & 376.623 \\
82.5 & 378.571 \\
82 & 380.145 \\
81.5 & 381.204 \\
81 & 381.734 \\
80.5 & 384.382
\end{tabular}




$\begin{array}{cc}80 & 385.971 \\ 79.5 & 387.249 \\ 79 & 388.287 \\ 78.5 & 390.363 \\ 78 & 393.477 \\ 77.5 & 395.552 \\ 77 & 400.396 \\ 76.5 & 405.931 \\ 76 & 412.85 \\ 75.5 & 423.757 \\ 75 & 438.105 \\ 74.5 & 461.631 \\ 74.3 & 489.307 \\ 74.1 & 529.785\end{array}$

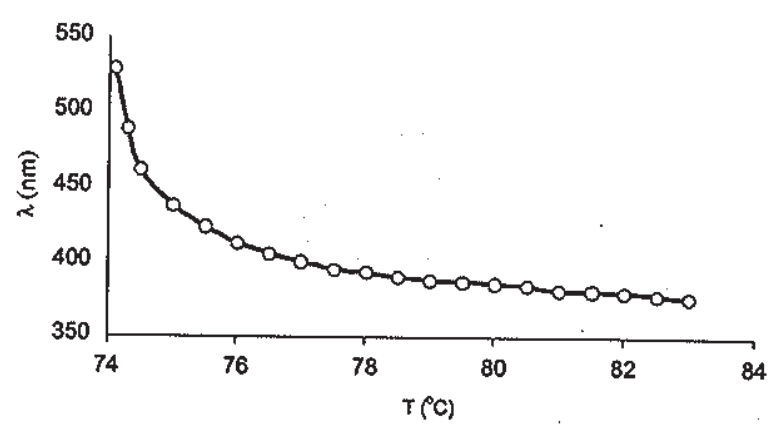

Figure 1.8(a): Dependence of the selective reflection wavelength on temperature for compound 1 .

Figure 1.8(a) shows the temperature dependence of $\lambda_{0}$ in the chiral nematic phase in the vicinity of the isotropic phase on the higher temperature side and the smectic A phase on the lower temperature side. Particularly interesting is the fact that on approaching the smectic A phase $\lambda_{0}$ diverges.

The chiral nematic - smectic $A$ is expected to show a strong pretransitional effect associated with the appearance of short range fluctuations of the smectic $A$ kind in the chiral nematic phase. Such behaviour is characteristic of true second order or weakly first order transitions. Specifically the divergence of $\lambda_{0}$ supports the concept 
mentioned earlier that the twist of the director is not permitted in the smectic A phase. In the absence of smectic like correlations, the pitch in the chiral nematic phase is expected to have an inverse temperature dependence, although generally weak (except in the vicinity of the transition to the isotropic phase). Figure $1.8(\mathrm{~b})$ shows the data collected away from the transition to the smectic A phase and we do see the expected behaviour. According to the anharmonic theory, the pitch $\mathrm{P} \propto 1 / \mathrm{T}$; a feature supported by the experimental dato in figure 1.8(b).

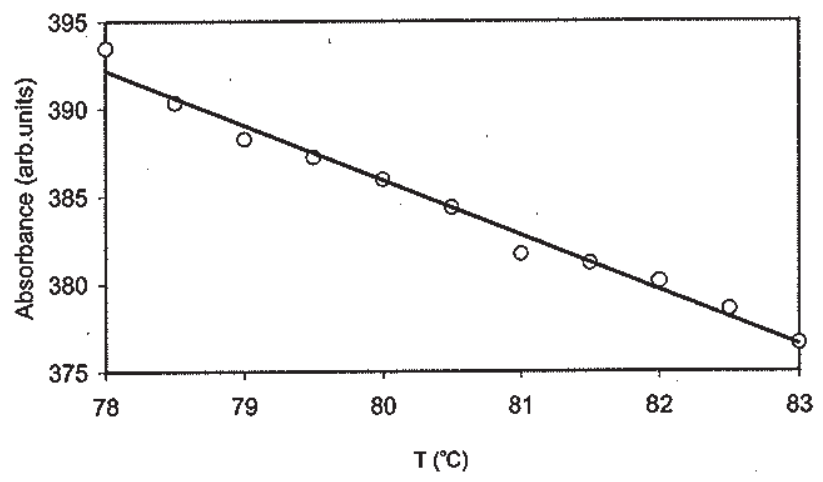

Figure 1.8 (b): Data of figure 3.8 (a) on an enlarged scale.

The pretransitional increase on approaching the smectic A phase has been analyzed in terms of the singular contribution coming from the twist elastic constant. To be precise,

$$
\mathrm{P}=\mathrm{P}_{0}+\delta \mathrm{K}_{22} \mathrm{P}_{0} / \mathrm{K}_{22}^{0}
$$

where $\mathrm{P}_{0}$ is the "background" pitch value and $\mathrm{K}_{22}^{0}$ is the twist elastic constant in the absence of any smectic correlations. The parameter of interest is $\delta \mathrm{K}_{22}$, which diverges on approaching the smectic A phase. From the analysis of parameters in the vicinity of a continuous (or second order) transition it has been shown that the temperature dependence of $\delta \mathrm{K}_{22}$ can be written as

$$
\delta \mathrm{K}_{22} \propto\left(\left(\mathrm{T}-\mathrm{T}_{\mathrm{c}}\right) / \mathrm{T}_{c}\right)^{\nu}
$$

where $T_{c}$ is the transition temperature and $v$ is the critical exponent connected with the growth of correlation length in the disordered phase (here chiral nematic). Determination of critical exponents like $v$ is very important from the viewpoint of understanding phase transitions in general. The tremendous amount of work, theoretical and experimental, that is taking place since the latter half of the last century has successfully brought in the concept of universality of phase transitions 
and the associated critical phenomena. The concept argues that the diverse phase transitions in all the condensed matter media can be grouped into "universality classes" based on just two parameters: the dimensions of the space (whether 3-d or 2-d) and the dimensionality of the order parameter; the microscopic details of the interactions between the entities (atoms or molecules) are unimportant. The success of this concept can be judged from the observation that diverse systems like ferromagnetic - paramagnetic, superfluid - normal fluid, superconducting normal, certain liquid crystalline transitions have been very well described using the expressions and exponents derived. According to this concept, the nematic (or chiral nematic) smectic A should belong to the "XY universality class" since the order parameter associated is a two component one being studied in a 3-dimensional space. This theory predicts the exponent $v$ to take a value of 0.67 . With this background we proceeded to fit the temperature - dependent pitch values shown in Table 1.1 and figure 1.8 and equation (1). We first described the non-singular variation seen away from the chiral nematic - smectic $A$ transition with a linear temperature dependent term (as a background) to take out the dependence on $P$. Then the singular past becomes essentially equation (2). Taking log on both sides to linearize the equation we get

$$
\log P \propto v \log \left(\left(T-T_{e}\right) / T_{c}\right)+\text { constant }
$$

the fit to equation (3) is shown in figure 1.9. As is generally true the singularity is valid close to the transition and therefore the linear behaviour close to the transition was used to calculate the slope, which is nothing but the exponent $v$. The determined $v$ value furns out to be $0.69 \pm 0.02$, which within the error bars agrees with the theoretical value mentioned above.

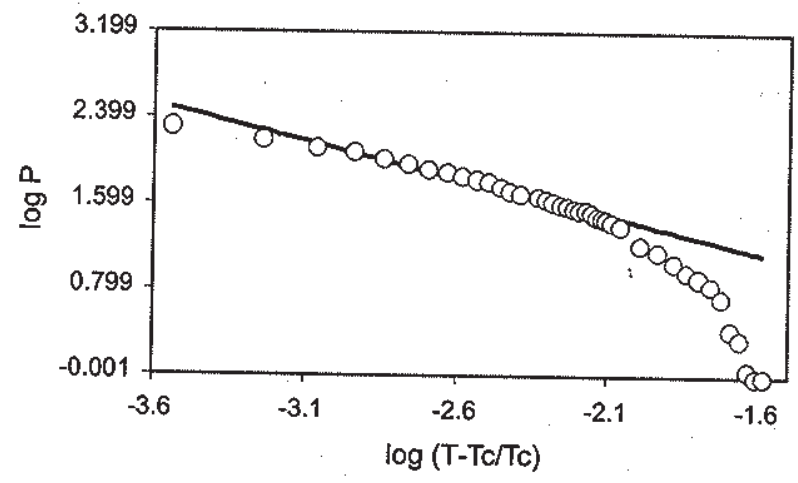

Figure 1.9: Graph showing log $P$ against $\log (\mathrm{T}-\mathrm{Tc} / \mathrm{TC})$ 


\section{Compound 2:}

Figures 1.10 and 1.11 shows the fextures observed under the polarizing microscope for the two mesophases immediately below the isotropic phase.

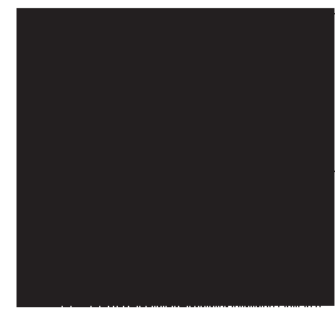

Figure 1.10: Texture seen is the chiral nematic phase of compound 2.

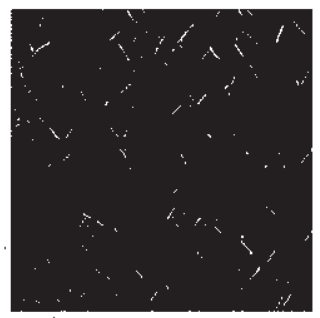

Figure 1.11: The filament texture characteristic of the $T G B_{A}$ phase seen in compound 2.

The filament texture seen in the figure 1.11 is characteristic of the TGB ${ }_{A}$ phase. The $X$-ray diffraction profiles taken in the chiral nematic and $T G B_{A}$ phases are produced in figure 1.12 .

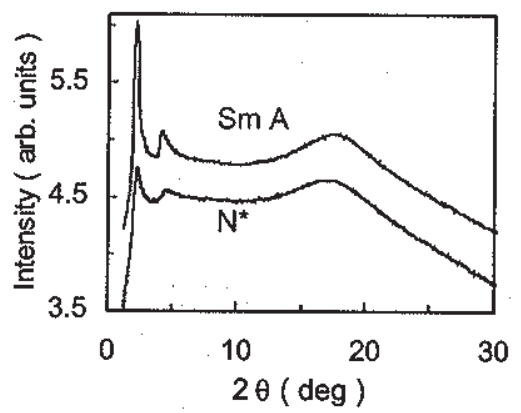

Figure 1.12: $X$-ray diffraction profiles in the chiral nematic and $T G B_{A}$ phases of compound 2. 
The fact that there is a broad diffuse peak at $2 \theta \sim 20^{\circ}$ suggests that both the phases are fluid in nature. The important feature to be noted, however, is that at low angles there are two sharp peaks which are intense in the TGB phase. This is the feature that indicates the presence of layered structure unlike that for the chiral nematic phase.

Typical spectroscopic profiles collected in the chiral nematic, $T G B_{A}$ as well as in the isotropic phases are shown in figure 1.13.

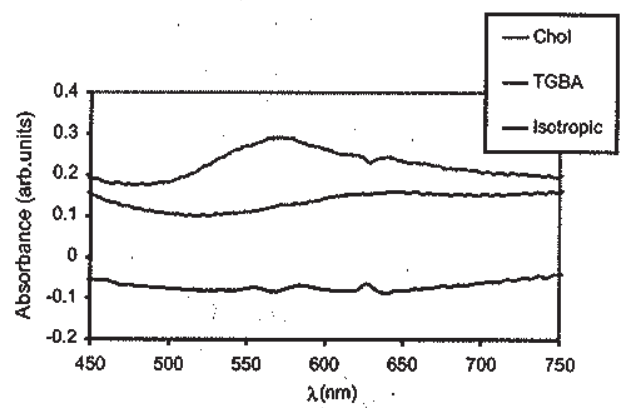

Figure 1.13: Spectroscopic profiles collected in the chiral nematic $(-)$, $T_{G B}(-)$ and isotropic $(-)$ phases.

The fact that the absorption peaks is strong and clear, together with an intense $X$-ray profile peak confirms the features expected for the $T G B_{A}$ phase.

The temperature dependence of the peak wavelength $\lambda_{0}$ determined from the spectroscopic profiles at intervals of $2^{\circ} \mathrm{C}$ is tabulated in table 1.2 and graphically represented in figure 1.14 .

Table 1.2: Temperature dependence of the selective reflection wavelength in the chiral nematic and $\mathrm{TGB}_{\mathrm{A}}$ phases of compound 2.

\begin{tabular}{cc}
\hline $\mathrm{T}\left({ }^{\circ} \mathrm{C}\right)$ & $\lambda(\mathrm{nm})$ \\
\hline 185 & 527.792 \\
183 & 539.221 \\
181 & 556.364 \\
179 & 558.961 \\
177 & 564.294 \\
175 & 567.187 \\
174 & 571.618
\end{tabular}




\begin{tabular}{lc}
173 & 573.533 \\
172 & 574.73 \\
171 & 576.884 \\
170 & 579.038 \\
169.5 & 582.149 \\
169 & 591.005 \\
168.5 & 598.904 \\
168 & 612.067 \\
167.5 & 615.369 \\
167 & 622.288 \\
166.5 & 624.451 \\
166 & 626.181 \\
165.5 & 626.613 \\
165 & 627.478 \\
\hline
\end{tabular}

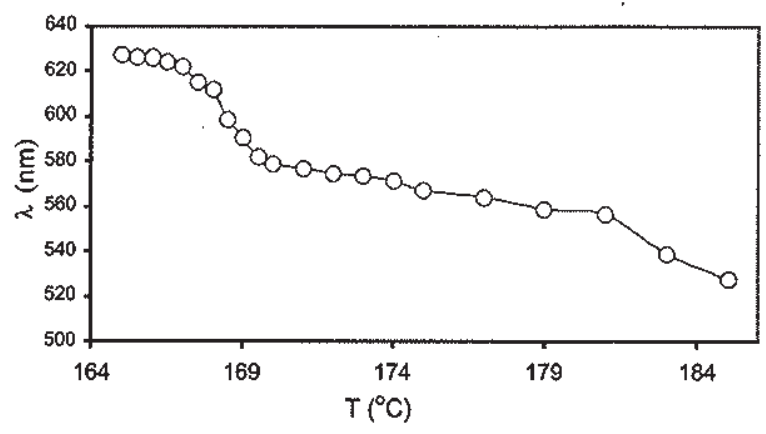

Figure 1.14: Selective reflection wavelength against temperature plot for compound 2.

Notice that there is a gradual increase in $\lambda_{0}$ with decreasing temperature in the temperature range $184-179^{\circ} \mathrm{C}$. This is owing to the proximity of the isotropic phase. But what is more striking is the abrupt increase followed by saturation in the value at the chiral nematic to $\mathrm{TGB}_{\mathrm{A}}$ transition. It may be mentioned that unlike in the case of chiral nematic to smectic $A$ where there was a true divergence (as seen for compound 1), here there is only an abrupt increase reaching a limiting value. The latter feature is important as it established the presence of a twisted structure in the phase. 


\section{Conclusion}

We have carried out selective reflection measurements as a function of temperature in two chiral liquid crystalline materials. The results clearly bring out the similarities and differences in the two compounds that arises from the different phase sequences they posses.

\section{References} 1. Hand Book of Liquid Crystal, D. Demus, J.W. Goodby, G.W. Gray, H.W. Spiess and V. Vill,
wiley-VCH, Germany (1998).

2. P S Pershan, Structure of Liquid Crystal Phases, World Scientific Publishing Co. Pvt. Ltd. 3. Heinz-Siegfried Kitzerow and Christian Bahr, Chirality in Liquid Crystals, Springer-verlag
New York. Inc.

4. P J Collings, Liquid Crystals: Natures Delicate Phase of Matter, Princeton University Press.

5. P.G. De Gennes and J. Prost, The Physics of Liquid Crystals, Oxford University Press.

6. Shri Singh, Liquid Crystals Fundamentals, World Scientific Publishing Co. Put. Ltd. 